\title{
Onset of Benard-Marangoni Ferroconvection with Internal Heat Generation
}

\author{
C. E. Nanjundappa $\cdot$ I. S. Shivakumara • \\ R. Arunkumar
}

Received: 21 October 2009 / Accepted: 18 June 2010 / Published online: 14 July 2010

(C) Springer Science+Business Media B.V. 2010

\begin{abstract}
The effect of internal heat generation on the onset of Benard-Marangoni convection in a horizontal ferrofluid layer heated from below in the presence of a uniform vertical magnetic field is studied. The lower boundary is rigid and the upper free boundary is assumed to be flat and undeformable. Both the boundaries are considered to be perfectly insulated to temperature perturbations and the resulting eigenvalue problem is solved numerically using the Galerkin technique as well as analytically by regular perturbation technique with wave number as a perturbation parameter. It is observed that the analytical results agree well with those obtained numerically. It is noted that the combined effect of magnetic Rayleigh number and dimensionless internal heat source strength is to reinforce together and to hasten the onset of BenardMarangoni ferroconvection compared to their presence in isolation. In addition, the nonlinearity of fluid magnetization is found to have no influence on the criterion for the onset of ferroconvection. Some existing results
\end{abstract}

C. E. Nanjundappa $(\varangle)$

Department of Mathematics,

Dr. Ambedkar Institute of Technology,

Bangalore 560 056, India

e-mail: cenanju@hotmail.com

I. S. Shivakumara $(\varangle)$

UGC-Centre for Advanced Studies in Fluid Mechanics,

Department of Mathematics, Bangalore University,

Bangalore 560 001, India

e-mail: shivakumarais@gmail.com

R. Arunkumar

Department of Mathematics,

Rajarajeswari College of Engineering,

Bangalore 560 074, India are reproduced as particular cases from the present study.

Keywords Benard-Marangoni ferroconvection • Ferrofluid - Galerkin technique •

Internal heat generation $\cdot$ Insulated boundaries

\section{Introduction}

Ferrofluids contain single domain nanoparticles of magnetic material stably suspended in a liquid carrier with low electrical conductivity. Each particle is encapsulated by a monolayer of surfactant in order to prevent particle coalescence due to magnetic attraction. The average size of magnetic nanoparticles is about $10 \mathrm{~nm}$. Magnetic colloids have magnetic susceptibility which is thousands times larger than that of natural materials. Such fluids are found to have promising potential for heat transfer applications in various areas of science, technology, and nanotechnology because they can be controlled by an external magnetic field (Rosensweig 1985; Bashtovoy et al. 1988, 1993).

The magnetization of ferromagnetic fluids depends on the magnetic field, the temperature and the density of the fluid. Any variation of these quantities can induce a change in body force distribution in the fluid. This leads to convection in ferrofluids in the presence of magnetic field gradient, known as ferroconvection, which is similar to buoyancy driven convection. Buoyancy driven convection in a layer of ferrofluid heated uniformly from below in the presence of a uniform magnetic field has attracted researchers due to its importance in heat transfer problems. The theory of convective instability in a horizontal layer of ferrofluid 
heated uniformly from below began with Finlayson (1970) and the topic covering various aspects has received increasing importance over the years (Lalas and Carmi 1971; Shliomis 1974; Gotoh and Yamada 1982; Stiles and Kagan 1990; Kaloni and Lou 2004). In his review article, Odenbach (2004) has focussed on recent developments in the field of rheological investigations of ferrofluids and their importance for the general treatment of ferrofluids. The nonlinear stability analysis for a magnetized ferrofluid layer heated from below has been performed by Sunil and Amit Mahjan (2008) for stress-free boundaries. Nanjundappa and Shivakumara (2008) have considered variety of velocity and temperature boundary conditions on the onset of ferroconvection in an initially quiescent ferrofluid layer. Thermal convection of ferrofluids in the presence of a uniform vertical magnetic field with the boundary temperatures modulated sinusoidally about some reference values is investigated by Singh and Bajaj (2009). Recently, the effect of magnetic field dependent (MFD) viscosity on the onset of convection in a ferromagnetic fluid layer heated from below in the presence of a vertical magnetic field has been investigated by considering the bounding surfaces are either rigid-ferromagnetic or stress-free with constant heat flux conditions (Nanjundappa et al. 2009).

Convection can also be induced by surface-tension forces provided it is a function of temperature. In view of the fact that heat transfer is greatly enhanced due to convection, the magnetic convection problems offer new possibilities for new applications in cooling with motors, loud speakers, transmission lines, and other equipment where magnetic field is already present. If the ferrofluid layer has an upper surface open to atmosphere then the instability is due to the combined effects of the buoyancy as well as temperaturedependent surface tension forces, known as BenardMarangoni ferroconvection. A limited number of studies have addressed the effect of surface tension forces on ferroconvection in a horizontal ferrofluid layer. Linear and non-linear stability of combined buoyancy-surface tension effects in a ferrofluid layer heated from below has been analyzed by Qin and Kaloni (1994). The linear stability analysis of a layer of magnetic fluid with deformable free surface which is heated uniformly from below and subject to a vertical magnetic field by Weilepp and Brand (1996) has been analyzed by considering the temperature dependence of the surface tension and buoyancy. The coupling between Marangoni and Rosensweig instabilities by considering two semi-infinite incompressible and immiscible viscous fluids of infinite lateral extent in which one of them is ferromagnetic and the other is a usual
Newtonian liquid is addressed by Weilepp and Brand (2001). The effect of different forms of basic temperature gradients on the onset of ferroconvection driven by combined surface tension and buoyancy forces has been discussed by Shivakumara et al. (2002), while Hennenberg et al. (2005) have discussed RayleighBenard-Marangoni instability in a ferrofluid layer in the presence of a weak vertical magnetic field normal to the boundaries. Shivakumara and Nanjundappa (2006) have analyzed the onset of Marangoni ferroconvection with different initial temperature gradients with the object of understanding control of convection. Recently, Bozhko and Putin (2009) have outlined the thermomagnetic convection in magnetic fluids under microgravity conditions.

The effect of quadratic basic state temperature gradient caused by uniform internal heat generation plays a decisive role in understanding control of convection. Copious literature is available on coupled BenardMarangoni convection in a horizontal ordinary viscous fluid layer with uniform distribution of internal heat generation (Char and Chiang 1994; Wilson 1997; Bachok and Arifin 2010 and references therein). Nonetheless, its counterpart in a ferrofluid layer has not received due attention in spite of its occurrence and importance in many technological applications which involve ferrofluids. In the present study, we have considered the problem of combined buoyancy and surface tension driven convection in a horizontal ferromagnetic fluid layer in the presence of uniform vertical magnetic field including the additional effect of internal heat generation. Such a study helps in understanding control of Benard-Marangoni ferroconvection due to non-uniform temperature gradient arising due to internal heating, which is important in the applications of ferrofluid technology. The lower rigid and upper free boundary at which the temperature-dependent surface tension forces are accounted for are considered to be perfectly insulated to temperature perturbations. The resulting eigenvalue problem is solved numerically using the Galerkin technique. Besides, an analytical formula is obtained for the critical Rayleigh/Marangoni number by regular perturbation technique with wave number as a perturbation parameter. The results obtained from both numerical and analytical methods are found to complement with each other suggesting the analytical results obtained are exact.

\section{Mathematical Formulation}

We consider a horizontal layer of an electrically nonconducting Boussinesq ferromagnetic fluid of thickness 
$d$ with a uniformly distributed volumetric heat generation. A uniform magnetic field $H_{0}$ is applied in the direction normal to the boundaries of the ferrofluid layer. The temperatures at the lower-rigid $(z=0)$ and upperfree $(z=\mathrm{d})$ boundaries are kept at $T_{l}$ and $T_{u}\left(<T_{l}\right)$, respectively. A Cartesian co-ordinate system $(x, y, z)$ is used with the origin at the bottom of the surface and the z-axis vertically upward. Gravity acts in the negative z-direction, $\vec{g}=-g \hat{k}$, where $\hat{k}$ is the unit vector in the $\mathrm{z}$-direction. For most of the fluids the capillary number is very small. Several investigators in the past have followed this assumption in the study of Marangoni problem and the free surface is assumed to be nondeformable (zero capillary number). In analyzing the problem it is postulated that the upper free surface is flat and the parametric values are well within the range of our basic assumption. At the upper free surface, the surface tension $\sigma$ is assumed to vary linearly with temperature in the form

$\sigma=\sigma_{0}-\sigma_{T}\left(T-T_{0}\right)$

where $\sigma_{0}$ is the unperturbed value and $-\sigma_{T}$ is the rate of change of surface tension with temperature $T$. The fluid density $\rho$ is assumed to vary linearly with temperature in the form

$\rho=\rho_{0}\left[1-\alpha_{t}\left(T-T_{0}\right)\right]$

where $\alpha_{t}$ is the thermal expansion coefficient and $\rho_{0}$ is the density at $T=T_{0}$.

The governing equations, in the Boussinesq approximation, are [1]:

$$
\nabla \cdot \vec{V}=0
$$

$$
\begin{aligned}
\rho_{0}\left[\frac{\partial \vec{V}}{\partial t}+(\vec{V} \cdot \nabla) \vec{V}\right]= & -\nabla \mathrm{p}+\rho \vec{g}+\mu_{0}(\vec{M} \cdot \nabla) \vec{H} \\
& +\mu \nabla^{2} \vec{V}
\end{aligned}
$$

$$
\begin{aligned}
& {\left[\rho_{0} C_{V, H}-\mu_{0} \vec{H} \cdot\left(\frac{\partial \vec{M}}{\partial T}\right)_{V, H}\right]} \\
& \times \frac{D T}{D t}+\mu_{0} T\left(\frac{\partial \vec{M}}{\partial T}\right)_{V, H} \cdot \frac{D \vec{H}}{D t}=k_{1} \nabla^{2} T+Q
\end{aligned}
$$$$
\nabla \cdot \vec{B}=0, \quad \nabla \times \vec{H}=0 \quad \text { or } \quad \vec{H}=\nabla \varphi
$$$$
\vec{B}=\mu_{0}(\vec{M}+\vec{H})
$$$$
\vec{M}=\left[M_{0}+\chi\left(H-H_{0}\right)-K\left(T-T_{0}\right)\right] \frac{\vec{H}}{H}
$$

where $\vec{V}=(u, v, w)$ is the velocity vector, $t$ the time, $p$ the pressure, $\vec{H}$ the magnetic field intensity, $\vec{M}$ the magnetization, $\vec{B}$ the magnetic induction, $\mu$ the dynamic viscosity, $\mu_{0}$ the magnetic permeability of vacuum, $k_{1}$ the thermal conductivity, $C_{V, H}$ the specific heat capacity at constant volume and magnetic field per unit mass, $Q$ the uniformly distributed volumetric heat generation within the ferrofluid layer, $\chi=(\partial M / \partial H)_{H_{0}, T_{0}}$ the magnetic susceptibility, $K=-(\partial M / \partial T)_{H_{0}, T_{0}}$ the pyromagnetic co-efficient, $\varphi$ the magnetic potential, $\nabla^{2}=\partial^{2} / \partial x^{2}+\partial^{2} / \partial y^{2}+\partial^{2} / \partial z^{2}$ the Laplacian operator, while $M_{0}=M\left(H_{0}, T_{0}\right), H=|\vec{H}|$ and $M=|\vec{M}|$.

The basic state is quiescent and is given by

$\vec{V}_{b}=0$

$$
\begin{aligned}
p_{b}(z)= & p_{0}-\rho_{0} g z-\rho_{0} \alpha_{t} g\left[\frac{Q z^{3}}{6 k_{1}}-\frac{Q d z^{2}}{4 k_{1}}+\frac{\beta z^{2}}{2}\right] \\
& -\frac{\mu_{0} M_{0} K}{1+\chi}\left[\frac{Q z^{2}}{2 k_{1}}-\frac{Q d z}{2 k_{1}}+\beta z\right]-\frac{\mu_{0} K^{2}}{(1+\chi)^{2}} \\
& \times\left[\frac{Q^{2} z^{4}}{8 k_{1}^{2}}+(2 \beta-Q d) \frac{Q z^{3}}{4 k_{1}}+\left(\beta-\frac{Q d}{2 k_{1}}\right) \frac{z^{2}}{2}\right]
\end{aligned}
$$

$T_{b}(z)=-\frac{Q z^{2}}{2 k_{1}}+\frac{Q d z}{2 k_{1}}-\beta z+T_{0}$

$$
\vec{H}_{b}(z)=\left[H_{0}-\frac{K}{1+\chi}\left(\frac{Q z^{2}}{2 k_{1}}-\frac{Q d z}{2 k_{1}}+\beta z\right)\right] \hat{k}
$$

$$
\vec{M}_{b}(z)=\left[M_{0}+\frac{K}{1+\chi}\left(\frac{Q z^{2}}{2 k_{1}}-\frac{Q d z}{2 k_{1}}+\beta z\right)\right] \hat{k}
$$

where $\beta=\left(T_{l}-T_{u}\right) / d=\Delta T / d$ and the subscript $b$ denotes the basic state. It may be noted that $T_{b}(z)$, $\vec{H}_{b}(z)$ and $\vec{M}_{b}(z)$ are distributed parabolically with the fluid layer height due to the presence of internal heat generation. However, for $Q=0$ (i.e. in the absence of internal heat generation) the basic state distributions are linear in $\mathrm{z}$.

To study the stability of the system, the basic state is perturbed in the form

$$
\begin{aligned}
\vec{V} & =\vec{V}^{\prime}, p=p_{b}(z)+p^{\prime}, \mathrm{T}=T_{b}(z)+T^{\prime}, \\
\vec{H} & =\vec{H}_{b}(z)+\vec{H}^{\prime}
\end{aligned}
$$


where the primed quantities represent the perturbed variables. Substituting Eq. 14 into Eq. 7, using Eqs. 6-8, we get (after dropping the primes):

$$
\begin{aligned}
& H_{x}+M_{x}=\left(1+M_{0} / H_{0}\right) H_{x} \\
& H_{y}+M_{y}=\left(1+M_{0} / H_{0}\right) H_{y} \\
& H_{z}+M_{z}=(1+\chi) H_{z}-K T
\end{aligned}
$$

where $\left(H_{x}, H_{y}, H_{z}\right)$ and $\left(M_{x}, M_{y}, M_{z}\right)$ are the $(x, y, z)$ components of the magnetic field intensity and magnetization, respectively. In obtaining the above equations it is assumed that $K \beta d<<(1+\chi) H_{0}$ and $K Q d^{2}<<$ $2(1+\chi) H_{0} k_{1}$.

Substituting Eq. 14 in 4, linearizing, eliminating the pressure by operating curl twice, and retaining the zcomponent of the resulting equation, we obtain (after dropping the primes):

$$
\begin{aligned}
\left(\rho_{0} \frac{\partial}{\partial t}-\mu \nabla^{2}\right) \nabla^{2} w= & -\mu_{0} K\left(-\frac{Q z}{k_{1}}+\frac{Q d}{2 k_{1}}-\beta\right) \\
& \times \frac{\partial}{\partial t}\left(\nabla_{h}^{2} \varphi\right)+\rho_{0} \alpha_{t} g \nabla_{h}^{2} T \\
& +\frac{\mu_{0} K^{2}}{1+\chi}\left(-\frac{Q z}{k_{1}}+\frac{Q d}{2 k_{1}}-\beta\right) \nabla_{h}^{2} T
\end{aligned}
$$

where $\nabla_{h}^{2}=\partial^{2} / \partial x^{2}+\partial^{2} / \partial y^{2}$ is the horizontal Laplacian operator.

Equation 5, after using Eq. 14, and linearizing, takes the form (after dropping the primes):

$$
\begin{aligned}
\frac{\partial T}{\partial t}-\mu_{0} T_{0} K \frac{\partial}{\partial t}\left(\frac{\partial \varphi}{\partial z}\right)= & k_{1} \nabla^{2} T+\left[1-\frac{\mu_{0} T_{0} K^{2}}{1+\chi}\right] \\
& \times\left(\frac{Q z}{k_{1}}-\frac{Q d}{2 k_{1}}+\beta\right) w
\end{aligned}
$$

where $\rho_{0} C_{0}=\rho_{0} C_{V, H}+\mu_{0} H_{0} K$ and we have assumed $\beta d<<T_{0}$.

Equation 6a,b, after substituting Eq. 14 and using Eq. 15, may be written as (after dropping the primes)

$$
\left(1+\frac{M_{0}}{H_{0}}\right) \nabla_{h}^{2} \varphi+(1+\chi) \frac{\partial^{2} \varphi}{\partial z^{2}}-K \frac{\partial T}{\partial z}=0 .
$$

The normal mode expansion of the dependent variables is assumed in the form

$$
\{w, T, \varphi\}=\{W(z), \Theta(z), \Phi(z)\} \exp [i(\ell x+m y)+\sigma t]
$$

where $\ell$ and $m$ are wave numbers in the $x$ and $y$ directions, respectively, and $\sigma$ is the growth rate which is complex.
On substituting Eq. 19 into Eqs. 16-18 and nondimensionalizing the variables by setting

$$
\begin{aligned}
& \left(x^{*}, y^{*}, z^{*}\right)=\left(\frac{x}{d}, \frac{y}{d}, \frac{z}{d}\right), W^{*}=\frac{d}{v} W, \Theta^{*}=\frac{\kappa}{\beta v d} \Theta, \\
& \Phi^{*}=\frac{(1+\chi) \kappa}{\mathrm{K} \beta v d^{2}} \Phi, t^{*}=\frac{v}{d^{2}} t
\end{aligned}
$$

where $v=\mu / \rho_{0}$ is the kinematic viscosity and $\kappa=$ $k_{1} / \rho_{0} C_{0}$ is the thermal diffusivity, we obtain (after ignoring the asterisks)

$$
\begin{aligned}
& {\left[\left(D^{2}-a^{2}\right)-\sigma\right]\left(D^{2}-a^{2}\right) W=}-R_{m} a^{2}\left[N_{S}(1-2 z)-1\right] \\
& \times(D \Phi-\Theta)+R_{t} a^{2} \Theta \\
&\left(D^{2}-a^{2}-\operatorname{Pr} \sigma\right) \Theta-\operatorname{Pr} M_{2} \sigma \Phi= {\left[N_{S}(1-2 z)-1\right] } \\
& \times\left(1-M_{2}\right) W \\
&\left(D^{2}-M_{3} a^{2}\right) \Phi=D \Theta .
\end{aligned}
$$

Here $D=d / d z$ is the differential operator, $a=\sqrt{\ell^{2}+m^{2}}$ is the overall horizontal wave number, $R_{t}=g \alpha_{t} \beta d^{4} / \nu \kappa$ is the thermal Rayleigh number, $M_{1}=$ $\mu_{0} K^{2} \beta /(1+\chi) \alpha_{t} \rho_{0} g$ is the magnetic number, $R_{m}=$ $R_{t} M_{1}=\mu_{0} K^{2} \beta^{2} d^{4} /(1+\chi) \mu \kappa$ is the magnetic Rayleigh number, $M_{2}=\mu_{0} T^{2} K^{2} /(1+\chi) \rho_{0} C_{0} \quad$ is the magnetic parameter, $M_{3}=\left(1+M_{0} / H_{0}\right) /(1+\chi)$ is the measure of nonlinearity of fluid magnetization parameter, $\operatorname{Pr}=v / \kappa$ is the Prandtl number and $N_{S}=Q d / 2 k_{1} \beta$ is the dimensionless heat source strength. As propounded by Vidal and Acrivos (1966) in the study of surface-tension driven convection problem, it is not possible to prove the principle of exchange of stability for the problem considered, in general. However, in discussing a similar problem, Weilepp and Brand (1996) have demonstrated through their numerical calculations that there is no oscillatory instability and convection sets in as stationary convection. On physical arguments also it is true that there is no mechanism to set up oscillatory motion. Based on these observations, the principle of exchange of stability is assumed to be valid for the problem considered. Moreover, the typical value of $M_{2}$ for magnetic fluids with different carrier liquids is of the order of $10^{-6}$ and hence its effect is neglected as compared to unity. Equations 21-23, after noting the above facts, now become

$$
\begin{aligned}
\left(D^{2}-a^{2}\right)^{2} W= & -R_{m} a^{2}\left[N_{S}(1-2 z)-1\right](D \Phi-\Theta) \\
& +R_{t} a^{2} \Theta
\end{aligned}
$$




$$
\left(D^{2}-a^{2}\right) \Theta=\left[N_{S}(1-2 z)-1\right] W
$$

$$
\left(D^{2}-M_{3} a^{2}\right) \Phi=D \Theta .
$$

It is considered that the lower boundary is rigidferromagnetic, while the upper free boundary at which the surface tension effects are accounted for is taken to be non-deformable and flat. In addition, both the boundaries are assumed to be perfectly insulated to temperature perturbations. The boundary conditions are then given by

$$
W=D W=\Phi=D \Theta=0 \quad \text { at } z=0
$$

$W=D^{2} W+M a a^{2} \Theta=D \Phi=D \Theta=0 \quad$ at $z=1$

where $M a=\sigma_{T} \Delta T d / \mu \kappa$ is the Marangoni number. It may be noted that the boundary conditions for the magnetic potential $\Phi$ considered are only the simpler ones. The assumption $D \Phi=0$ on the top free surface was used by Finlayson (1970) in his discussion of the convective instability problem in a ferrofluid layer for the free boundaries. This corresponds to the case, where the magnetic susceptibility is very high (i.e., $\chi \rightarrow \infty$ ). The assumption $\Phi=0$ on the bottom rigid boundary was used by Gotoh and Yamada (1982). This is a special case which can be attained when the magnetic permeability of the solid boundary is much higher than the magnetic permeability of the fluid.

\section{Method of Solution}

Equations 24-26 together with the boundary conditions constitute an eigenvalue problem with $R_{t}$ or $M a$ as an eigenvalue. The eigenvalue problem is solved both numerically using the Galerkin method as well as analytically using a regular perturbation technique with wave number as a perturbation parameter.

\section{Solution by Galerkin Technique}

The Galerkin method is used to solve the eigenvalue problem as explained in the book by Finlayson (1972). In this method, the test (weighted) functions are the same as the base (trial) functions. Accordingly, $W, \Theta$ and $\Phi$ are written as

$$
\begin{aligned}
& W(z)=\sum_{i=1}^{n} A_{i} W_{i}(z), \quad \Theta(z)=\sum_{i=1}^{n} B_{i} \Theta_{i}(z) \text { and } \\
& \Phi(z)=\sum_{i=1}^{n} C_{i} \Phi_{i}(z)
\end{aligned}
$$

where $A_{i}, B_{i}$ and $C_{i}$ are unknown constants to be determined. The base functions $W_{i}(z), \Theta_{i}(z)$ and $\Phi_{i}(z)$ are generally chosen such that they satisfy the corresponding boundary conditions but not the differential equations. We select the trial functions as

$W_{i}=\left(z^{4}-5 z^{3} / 2+3 z^{2} / 2\right) T_{i-1}^{*}, \Theta_{i}=z(1-z / 2) T_{i-1}^{*}$,

$\Phi_{i}=z^{2}(1-2 z / 3) T_{i-1}^{*}$

where $T_{i}^{* \prime} s$ are the modified Chebyshev polynomials. The above trial functions satisfy all the boundary conditions except the natural one, namely $D^{2} W+$ $M a a^{2} \Theta=0$ at $z=1$ but the residual from this condition is included as residual from the differential equation. Substituting Eq. 29 into 24-26, multiplying momentum Eq. 24 by $W_{j}(z)$, energy Eq. 25 by $\Theta_{j}(z)$ and magnetization Eq. 26 by $\Phi_{j}(z)$; performing the integration by parts with respect to $\mathrm{z}$ between $\mathrm{z}=0$ and $\mathrm{z}=1$ and using the boundary conditions, we obtain a system of linear homogeneous algebraic equations in $A_{i}, B_{i}$ and $C_{i}$. A nontrivial solution to the system requires the characteristic determinant of the coefficient matrix must vanish and this leads to a relation involving the parameters $R_{t}, M a, R_{m}, M_{1}, M_{3}, N_{s}$ and $a$ in the form

$f\left(R_{t}, M a, R_{m}, M_{1}, M_{3}, N_{s}, a\right)=0$.

The critical values of $R_{t c}$ or $M a_{c}$ are found as a function of wave number $a$ for various values of physical parameters. It is observed that the convergence is achieved with six terms in the series expansion of Eq. 29.

\section{Solution by Regular Perturbation Technique}

Since the critical wave number is negligibly small when the boundaries are perfectly insulated to temperature perturbations (i.e., $D \Theta=0$ at $z=0,1$ ), the eigenvalue problem is also solved analytically using regular perturbation technique with wave number $a$ as a perturbation parameter. Accordingly, the variables $W, \Theta$ and $\Phi$ are expanded in powers of $a^{2}$ as

$$
(W, \Theta, \Phi)=\left(W_{0}, \Theta_{0}, \Phi_{0}\right)+a^{2}\left(W_{1}, \Theta_{1}, \Phi_{1}\right)+\cdots .
$$


Substituting Eq. 31 into Eqs. 24-26 and also in the boundary conditions, and collecting the terms of zeroth order, we obtain

$$
D^{4} W_{0}=0
$$

$D^{2} \Theta_{0}=-W_{0}$

$D^{2} \Phi_{0}=-D \Theta_{0}$

with the boundary conditions

$W_{0}=D W_{0}=0=D \Theta_{0}=\Phi_{0} \quad$ at $z=0$

$W_{0}=D^{2} W_{0}=0=D \Theta_{0}=D \Phi_{0} \quad$ at $z=1$.

The solution to the zero-th order equations is found to be

$W_{0}=0, \Theta_{0}=1$ and $\Phi_{0}=0$.

The first order equations are then

$D^{4} W_{1}=R_{t}-R_{m}\left[N_{s}(1-2 z)-1\right]$

$D^{2} \Theta_{1}=1+W_{1}\left[N_{s}(1-2 z)-1\right]$

$D^{2} \Phi_{1}=D \Theta_{1}$

with the boundary conditions

$W 1_{1}=D W_{1}=\Phi_{1}=D \Theta_{1}=0 \quad$ at $z=0$

$W_{1}=D^{2} W_{1}+M a=D \Phi_{1}=D \Theta_{1}=0 \quad$ at $z=1$.

The general solution of Eq. 35a is given by

$W_{1}=c_{1} z^{2}+c_{2} z^{3}+c_{3} z^{4}+c_{4} z^{5}$

where

$c_{1}=\frac{R_{t}+R_{m}}{16}-\frac{R_{m} N_{s}}{240}+\frac{M a}{4}$

$c_{2}=-\frac{5\left(R_{t}+R_{m}\right)}{48}+\frac{7 R_{m} N_{s}}{240}-\frac{M a}{4}$

$c_{3}=\frac{R_{t}+R_{m}}{24}-\frac{R_{m} N_{s}}{24}$

$c_{4}=\frac{R_{m} N_{s}}{60}$.
From Eq. 35b, after using the condition that $D \Theta_{1}=0$ at $\mathrm{z}=0$ and $\mathrm{z}=1$, it follows that

$1=\int_{0}^{1}\left[1-N_{s}(1-2 z)\right] W_{1} d z$.

Substituting for $W_{1}$ from Eq. 37 into Eq. 38 and carrying out the integration leads to an expression of the form

$$
\begin{aligned}
& \frac{R_{t c}+R_{m}}{320}+\frac{M a_{c}}{48}+\frac{R_{m} N_{s}}{2880}+N_{s} \\
& \quad \times\left[\frac{R_{t c}+R_{m}}{2880}+\frac{M a_{c}}{240}+\frac{11 R_{m} N_{s}}{100800}\right]=1 .
\end{aligned}
$$

From Eq. 39 it is interesting to note that the parameter $M_{3}$ is not appearing in the expression and hence the nonlinearity of magnetization has no effect on the onset of Benard-Marangoni ferroconvection. Since at the onset of convection $a_{c}=0$ (very large wave length), one would expect that $M_{3}$ has no effect on the stability of the system. Besides, it can be seen that the parameters $M_{1}$ and $M_{3}$ have no influence on the onset of pure Marangoni ferroconvection $\left(R_{t}=0\right)$ in the absence of internal heat generation $\left(N_{s}=0\right)$. The numerical calculations carried out in the previous section also reflected the same behavior.

It is interesting to check Eq. 39 under the limiting conditions. When we set $R_{m}=0$ and $N_{s}=0$, Eq. 39 reduces to

$\frac{R_{t c}}{320}+\frac{M a_{c}}{48}=1$

a known result for ordinary viscous fluid layer (GarciaYbarra et al. 1987; Yang and Yang 1990).

When $M a_{c}=0$ and $N_{s}=0$, Eq. 39 reduces to

$R_{t c}=\frac{320}{\left(1+M_{1}\right)}$

and coincides with the result obtained by Nanjundappa and Shivakumara (2008).

When we set $R_{t c}=0$ and $R_{m}=0$, Eq. 39 reduces to

$M a_{c}=\frac{240}{5+N_{s}}$

which corresponds to the result obtained by Wilson (1997).

When $N_{s}=0$, Eq. 42 simply reduces to $M a_{c}=48$ and this is the known exact value for the clear viscous fluid layer (Pearson 1958). 
Fig. 1 Critical Rayleigh number $R_{t c}$ versus internal heat generation $N_{s}$ for different values of $R_{m}$

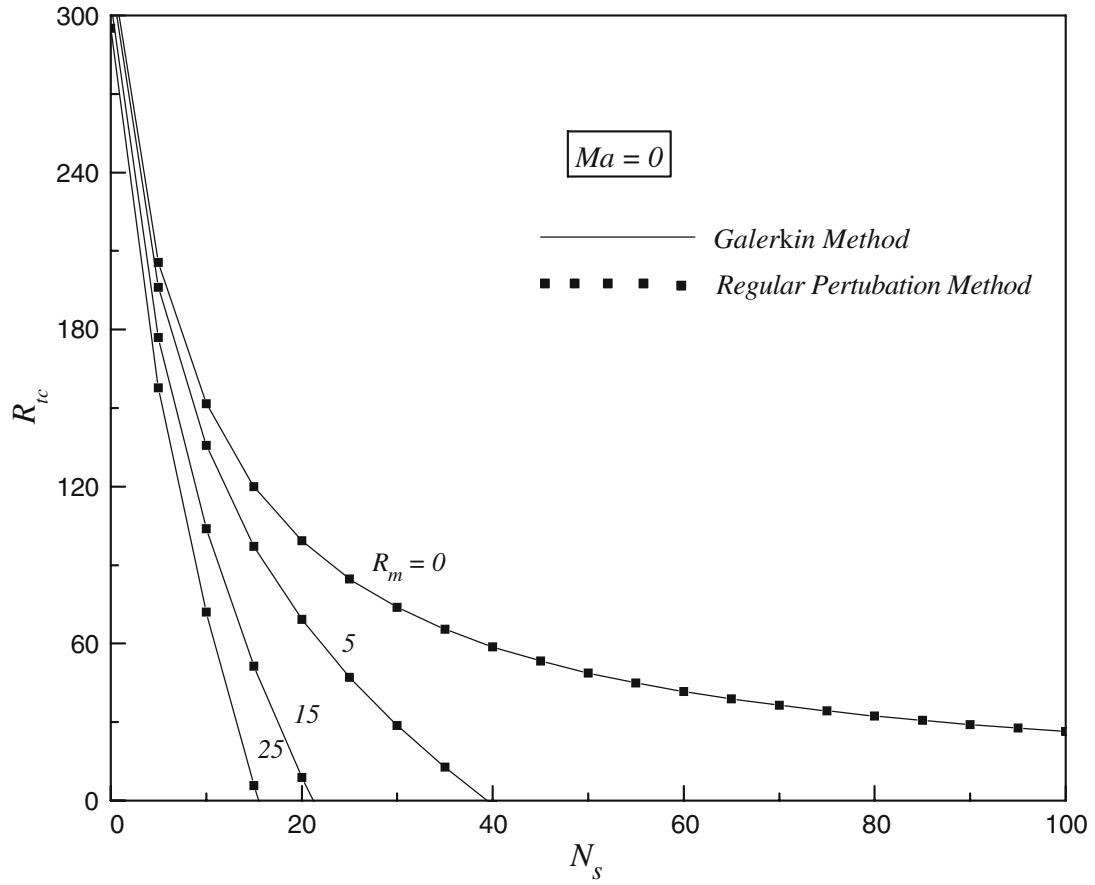

\section{Results and Discussion}

The linear stability analysis is carried out to investigate the effect of internal heat generation on the onset of coupled Benard-Marangoni convection in a horizontal ferrofluid layer in the presence of a uniform vertical magnetic field. The boundaries are assumed to be perfectly insulated to temperature perturbations and the critical eigenvalue $\left(M a_{c}\right.$ or $\left.R_{t c}\right)$ and the corresponding wave number $\left(a_{c}\right)$ are computed numerically by the Galerkin technique as well as analytically by employing a regular perturbation technique for different values of $R_{m}, M_{1}$ and $N_{s}$. The salient characteristics of these parameters on the stability of the system are exhibited graphically in Figs. 1, 2, 3 and 4. In these figures, the results obtained from the above two techniques are
Fig. 2 Critical Marangoni number $M a_{c}$ versus internal heat generation $N_{s}$ for different values of $R_{m}$

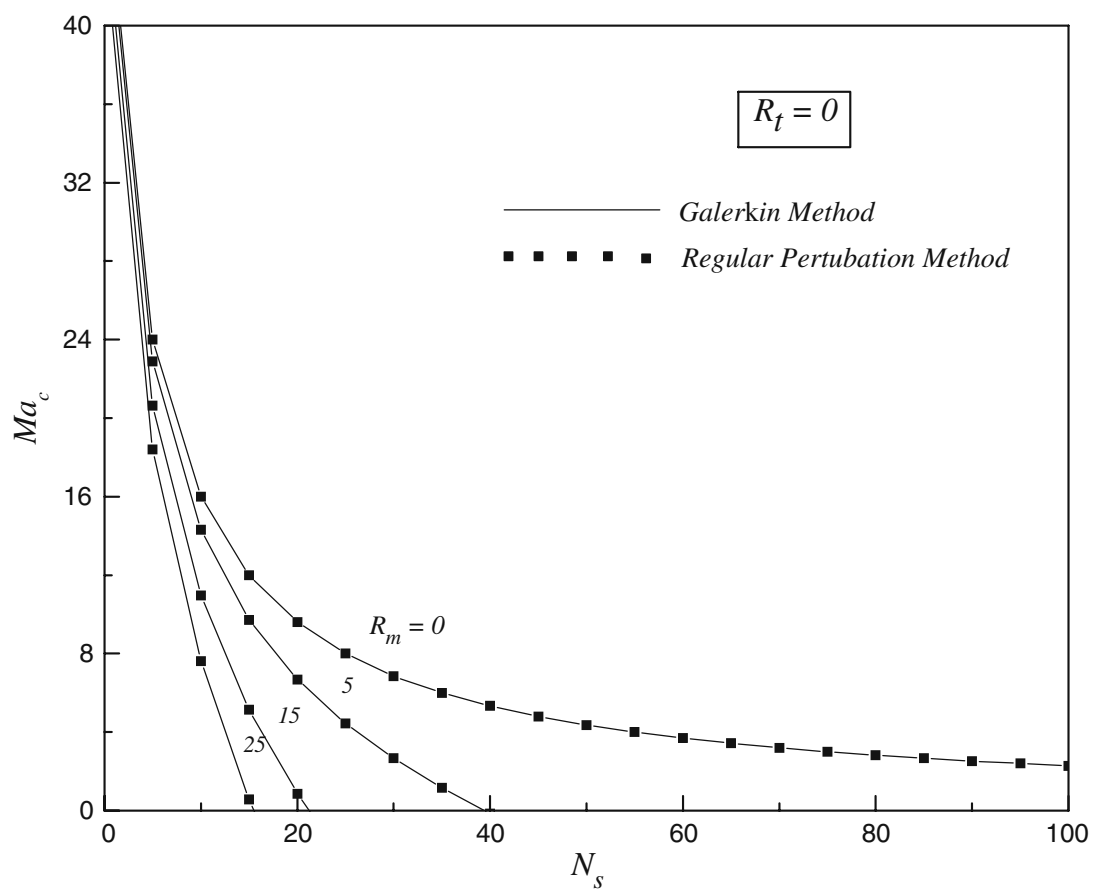


Fig. 3 Plot of locus of critical Rayleigh number $R_{t c}$ versus critical Marangoni number $M a_{c}$ for different values of $M_{1}$ for $N_{s}=5$

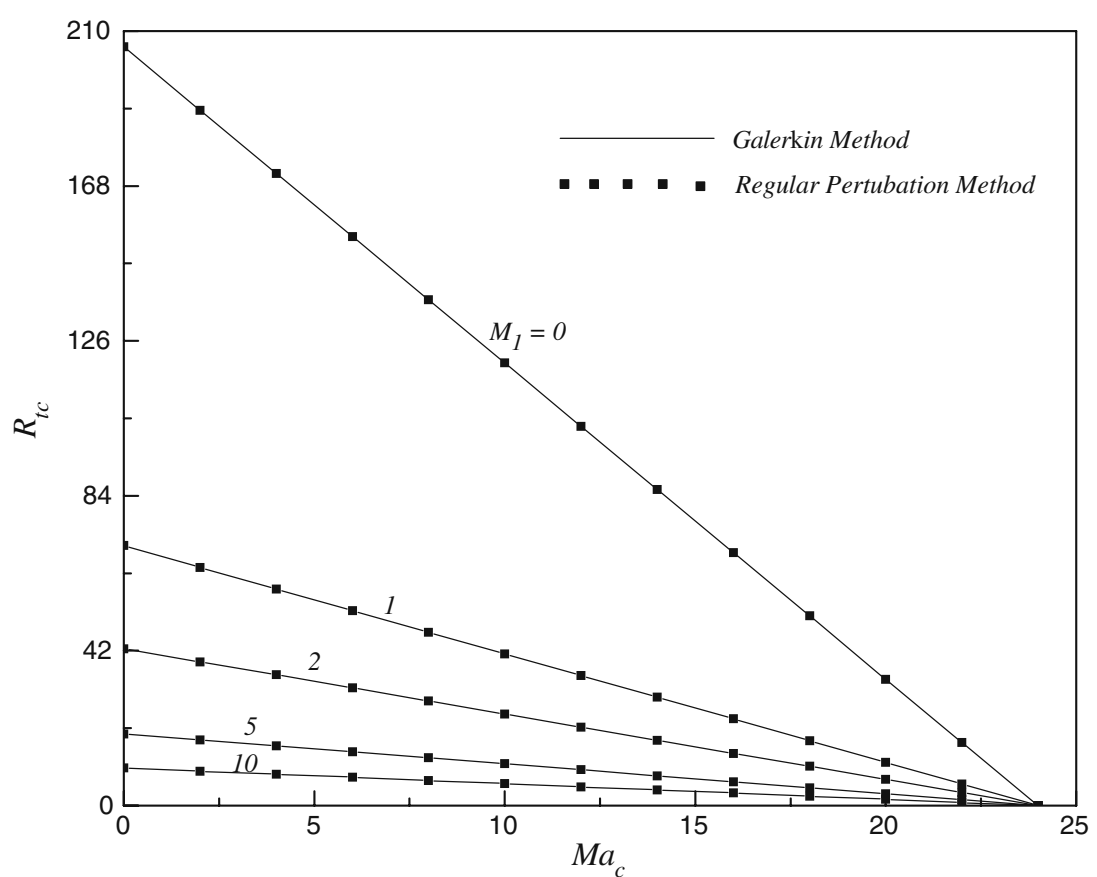

compared. In general, it is seen that the results obtained by regular perturbation technique coincide exactly with those obtained numerically and thus provides a justification for the use of regular perturbation technique in solving the eigenvalue problem when the boundaries are insulating to temperature perturbations. In the present context, we affirm that the analytical results obtained for the present case are exact.
Figure 1 shows the variation of critical thermal Rayleigh number $R_{t c}$ as a function of dimensionless internal heat source strength $N_{s}$ for different values of magnetic Rayleigh number $R_{m}$ when $M a=0$. This case corresponds to convective instability only due to buoyancy forces. The figure clearly indicates that $R_{t c}$ decreases monotonically with $N_{s}$ indicating the effect of increasing internal heating is to destabilize the sys-
Fig. 4 Plot of locus of critical Rayleigh number $R_{t c}$ versus critical Marangoni number $M a_{c}$ for different values of $N_{s}$ for $M_{1}=2$

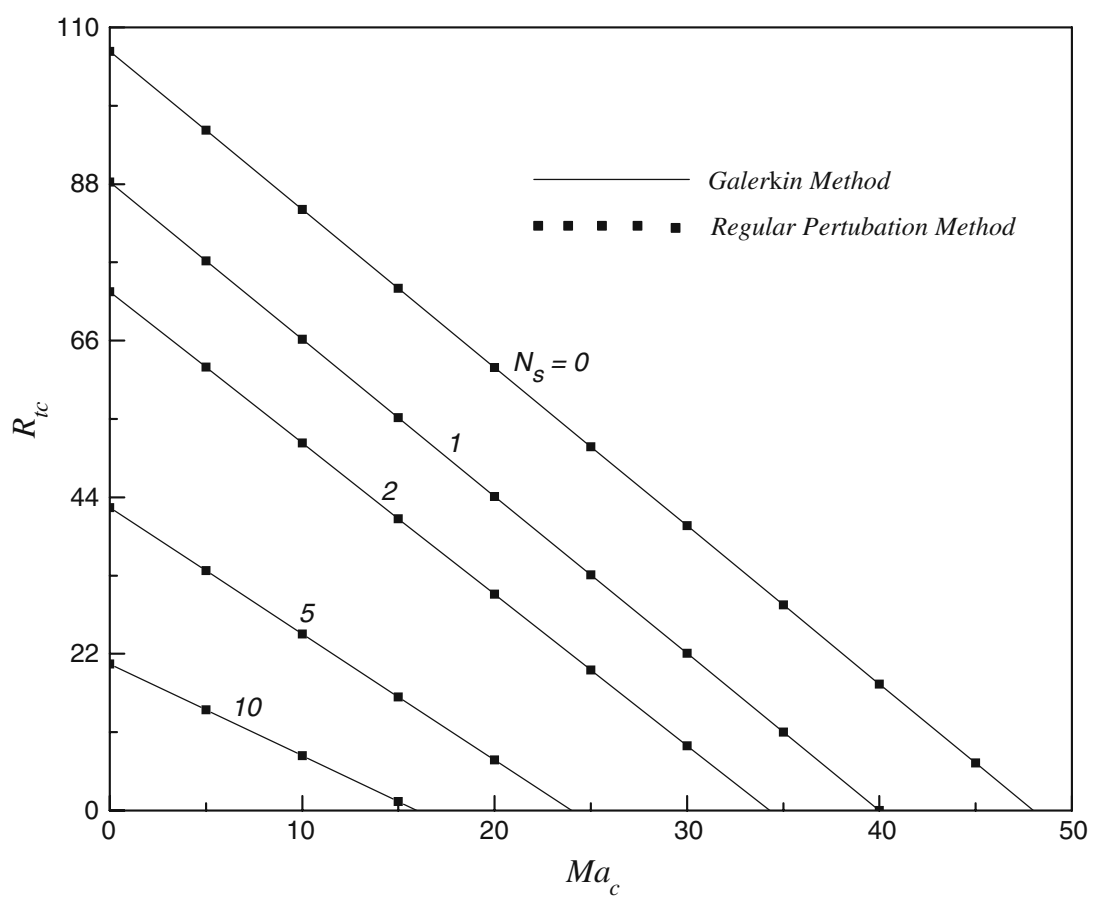


Fig. 5 Vertical velocity eigenfunction for different values of $M_{1}$ for $N_{s}=5$

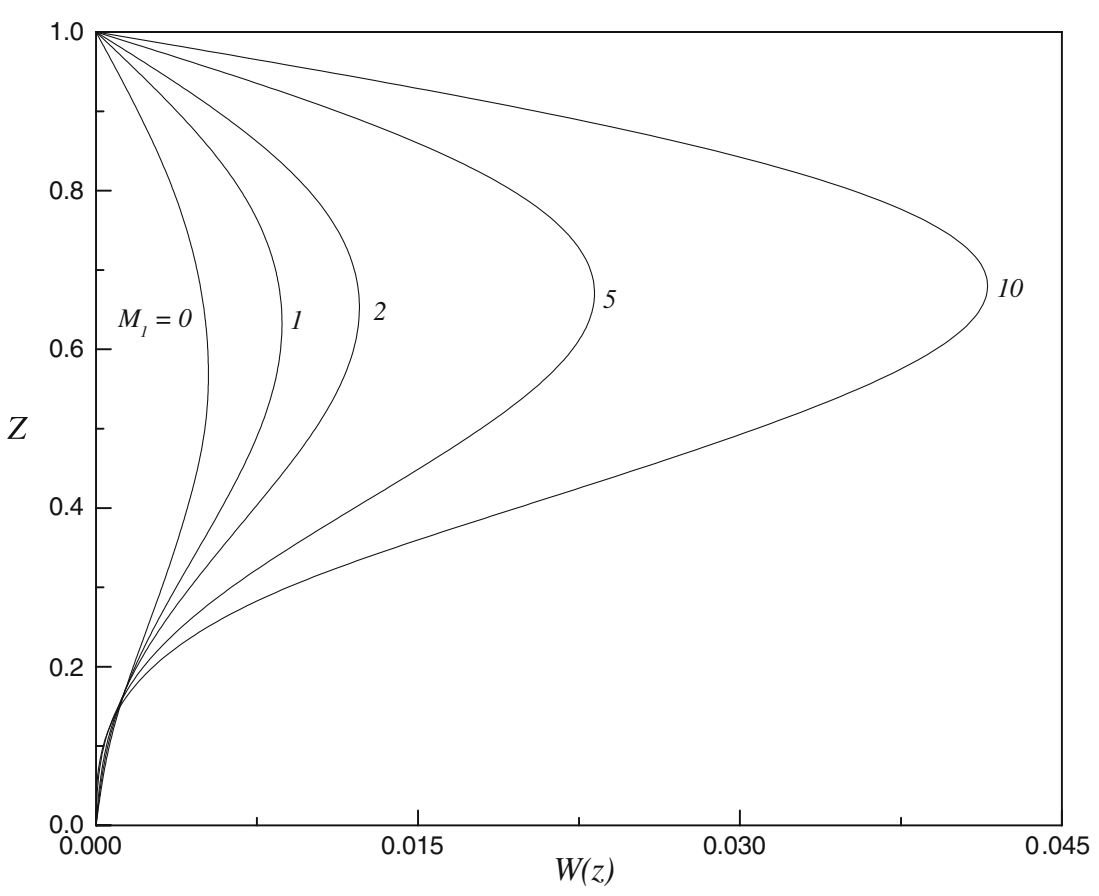

tem. This is because increasing $N_{s}$ amounts to large deviation in the basic temperature distribution of the parabolic type which in turn enhances the thermal disturbances in the fluid layer. It is more so with an increase in the value of $R_{m}$ and this is due to additive reinforcement of destabilizing magnetic force. In the case of increasing $R_{m}$, it is seen that $R_{t c}$ decreases quite rapidly first and then quite slowly. Thus the combined effect of internal heating and magnetic force is to reinforce together and to hasten the onset of ferroconvection compared to their effect in isolation. A similar situation prevails in the absence of thermal buoyancy forces (i.e. $R_{t}=0$ ) and this case corresponds to Marangoni ferroconvection (see Fig. 2).

We look into the simultaneous presence of thermal buoyancy and surface tension forces on the stability of the system. A plot of critical Rayleigh number $R_{t c}$ as a function critical Marangoni number $M a_{c}$ is shown in
Fig. 6 Vertical velocity eigenfunction for different values of $N_{s}$ for $M_{1}=2$

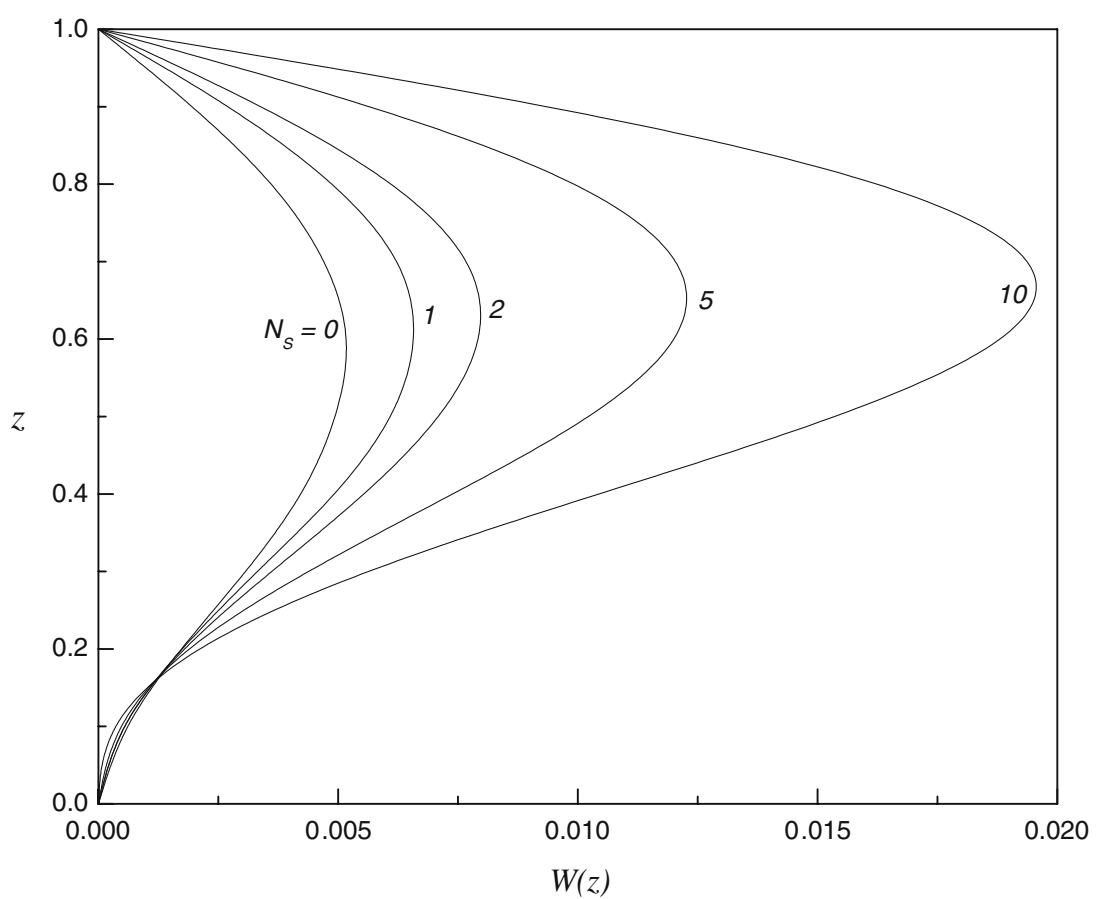


Fig. 3 for different values of $M_{1}$ with $N_{s}=5$ and in Fig. 4 for different values of $N_{s}$ with $M_{1}=2$. From the figures it is obvious that there is a strong coupling between the critical Rayleigh and the Marangoni numbers. That is, when the buoyancy force is predominant the surface tension force becomes negligible and viceversa. From Fig. 3 it is seen that an increase in the value of $M_{1}$ is to decrease the value of $R_{t c}$ and thus its effect is to hasten the onset of convection. This is attributed to the increase in destabilizing magnetic force. Nonetheless, the curves of different $M_{1}$ converge to the same value $M a_{c}=24$ when $R_{t c}=0$ indicating that it has no effect on Marangoni ferroconvection. The analytically obtained results also corroborate this behavior (see Eq. 4). Figure 4 shows that increase in the value of $N_{s}$ is to decrease both $M a_{c}$ and $R_{t c}$. Thus the non-linear temperature distributions arising due to volumetric distribution of heat sources is to advance the onset of ferroconvection. It is further observed that the critical Rayleigh/Marangoni numbers are independent of the nonlinearity of fluid magnetization parameter $M_{3}$ and the analytically obtained results also confirm this finding (see Eq. 39).

The perturbed vertical velocity eigenfunction $W(z)$ is presented in Figs. 5 and 6 for different values of $M_{1}$ and $N_{s}$, respectively. As can be seen, increase in the value of $M_{1}$ (see Fig. 5) and $N_{s}$ (see Fig. 6) is to drive the flow with more vigor and thus their effect is to hasten the onset of ferroconvection.

\section{Conclusions}

The effect of internal heat generation on the onset of coupled Benard-Marangoni ferroconvection is studied theoretically for perfectly insulated boundaries. From the foregoing study, the following conclusions can be drawn:

1. The effect of increase in the value of magnetic Rayleigh number $R_{m}$ and dimensionless internal heat source strength $N_{s}$ is to reinforce together and to hasten the onset of coupled Benard-Marangoni ferroconvection.

2. The nonlinearity of fluid magnetization parameter $M_{3}$ has no effect on the onset of ferroconvection.

3. The buoyancy and surface tension forces complement with each other and it is always found that $M a_{c}<R_{t c}$; a result in accordance with ordinary viscous fluids.

4. The magnetic number $M_{1}$ has no effect on the onset of pure Marangoni ferroconvection in the absence of internal heat generation but otherwise its effect is to advance the onset of convection.

5. The critical eigenvalues obtained by a regular perturbation technique and numerically by the Galerkin technique complement with each other indicating the analytical solutions obtained are exact

Acknowledgements The work reported in this paper was supported by UGC under CAS Programme. The authors (CEN) and (RA) wish to thank respectively, the Management and Principal of Dr. Ambedkar Institute of Technology and Rajarajeswari College of Engineering, Bangalore for the encouragement. The authors wish to thank the reviewers for their useful comments and suggestions.

\section{References}

Bachok, N., Arifin, N.M.: Feedback control of the MarangoniBenard convection in a horizontal fluid layer with internal heat generation. Microgravity Sci. Technol. 22, 97-105. doi:10.1007/s12217-009-9129-5 (2010)

Bashtovoy, V.G., Berkovsky, B.M., Vislovich, A.N.: Introduction to Thermomechanics of Magneticfluids. Hemisphere, Washington (1988)

Bashtovoy, V.G., Berkovsky, B.M., Vislovich, A.N.: Magnetic Fluids Engineering Applications. Oxford University Press, Oxford (1993)

Bozhko, A., Putin, G.: Thermomagnetic convection as a tool for heat and mass transfer control in nanosize materials under microgravity conditions. Microgravity Sci. Technol. 21, 8993 (2009)

Char, M.I., Chiang, K.T.: Stability analysis of Benard-Marangoni convection in fluids with internal heat generation. J. Phys., D, Appl. Phys. 27, 748-755 (1994)

Finlayson, B.A.: Convective instability of ferromagnetic fluids. J. Fluid Mech. 40, 753-767 (1970)

Finlayson, B.A.: Method of Weighted Residuals and Variational Principles. Academic Press, London (1972)

Garcia-Ybarra, P.L., Gastillo, J.L., Valerde, M.G.: BenardMarangoni convection with deformable interface and poorly conducting boundaries. Phys. Fluids 30, 2655-2661 (1987)

Gotoh, K., Yamada, M.: Thermal convection in a horizontal layer of magnetic fluids. J. Phys. Soc. Jpn. 51, 3042-3048 (1982)

Hennenberg, M., Weyssow, B., Slavtchev, S., Alexandrov, V., Desaive, T.: Rayleigh-Marangoni-Benard instability of a ferrofluid layer in a vertical magnetic field. J. Magn. Magn. Mater 289, 268-271 (2005)

Kaloni, P.N., Lou, J.X.: Convective instability of magnetic fluids under alternating magnetic fields. Phys. Rev. E 70, 0663113026324 (2004)

Lalas, D.P., Carmi, S.: Thermoconvective stability of ferrofluids. Phys. Fluids 4, 436-437 (1971)

Nanjundappa, C.E., Shivakumara, I.S.: Effect of velocity and temperature boundary conditions on convective instability in a ferrofluid layer. ASME J. Heat Transfer 130, 10450211045025 (2008)

Nanjundappa, C.E., Shivakumara, I.S., Srikumar, K.: Effect of MFD viscosity on the onset of ferromagnetic fluids layer heated from below and cooled from above with constant heat flux. Meas. Sci. Rev. 9(3), 77-78 (2009) 
Odenbach, S.: Recent progress in magnetic fluid research. J. Phys., Condens. Matter 16, 1135-1150 (2004)

Pearson, J.R.A.: On convection cells induce by surface tension. J. Fluid Mech. 4, 489-500 (1958)

Qin, Y., Kaloni, P.N.: Nonlinear stability problem of a ferromagnetic fluid with surface tension effect. Eur. J. Mech. B, Fluids 13, 305-321 (1994)

Rosensweig, R.E.: Ferrohydrodynamics. Cambridge University Press, New York (1985)

Shivakumara, I.S., Nanjundappa, C.E.: Marangoni ferroconvection with different initial temperature gradients. J. Energy Heat Mass Transf. 28, 45-61 (2006)

Shivakumara, I.S., Rudraiah, N., Nanjundappa, C.E.: Effect of non-uniform basic temperature gradient on RayleighBenard-Marangoni convection in ferrofluids. J. Magn. Magn. Mater. 248, 379-395 (2002)

Shliomis, M.I.: Magnetic fluids. Soviet Phys., Uspekhi (Engl. Trans.) 17, 153-169 (1974)

Singh, J., Bajaj, R.: Temperature modulation in ferrofluid convection. Phys. Fluids 21, 0641051-06410512 (2009)
Stiles, P.J., Kagan, M.: Thermoconvective instability of a ferrofluid in a strong magnetic field. J. Colloid Interface Sci. 134, 435-448 (1990)

Sunil, Amit Mahjan.: A nonlinear stability analysis for magnetized ferrofluid heated from below. Proc. R. Soc. Lond., A Math. Phys. Eng. Sci. 464, 83-98 (2008)

Vidal, A., Acrivos, A.: Nature of the neutral state in surfacetension driven convection. Phys. Fluids 9, 615-616 (1966)

Weilepp, J., Brand, H.R.: Competition between the BenardMarangoni and the Rosensweig instability in magnetic fluids. J. Phys. II France 6, 419-441 (1996)

Weilepp, J., Brand, H.R.: Coupling between Marangoni and Rosensweig instabilities. Part I: the transfer wave. Eur. Phys. J., Appl. Phys. 16, 217-229 (2001)

Wilson, S.K.: The effect of uniform internal heat generation on the onset of steady Marangoni convection in a horizontal layer of fluid. Acta Mech. 124, 63-68 (1997)

Yang, H.Q., Yang, K.T.: Benard-Marangoni instability in a two layer system with uniform heat flux. J. Thermophys. 4, 73-78 (1990) 